\title{
Behavior of Propylene Glycol (PG) in Dermis after Treatment of Rat Intact Skin Surface with Fatty Acids, Fatty Amines or Azone Dissolved in PG
}

\author{
Yoshikazu Takeuchi, Hidehito Yasukawa, Yumiko Yamaoka, Kenji TaGUCHI, \\ Shoji Fukushima, Yasuhiro Shimonaka, Hiroko Nishinaga, and Yasuko Morimoto \\ Department of Pharmaceutics, Faculty of Pharmaceutical Sciences, Kobe Gakuin University, Arise Igawadani-cho, \\ Nishi-ku, Kobe 651-21, Japan. Received June 6, 1994; accepted October 13, 1994
}

\begin{abstract}
Rat abdominal intact skin was treated with fatty acids, fatty amines, or Azone which were dissolved in propylene glycol (PG) and PG appearing in the rat dermis was studied. Analysis was done by Fourier transform infrared/attenuated total reflection (FT-IR/ATR) spectroscopy. The appearance of PG with time seemed to be in three phases when the skin sample was treated with a skin penetration enhancer such as oleic acid: (1) in the first stage, PG penetrated the skin barrier which was not substantially altered, and gradually appeared in the dermis; (2) in the second stage, it rapidly distributed in/throughout the dermis, and this rapid distribution was probably due to the alteration of the dermal structure: the penetration enhancing effect of the enhancer was thought to reach maximal; and (3) in the third stage, PG was saturated in the dermis. The value of $T_{\max \text { alteration }}$, at which the alteration of the dermal structures is completed, showed that the action of both oleic acid and oleylamine were more rapid than other enhancers. Both the value of PG peak area $a_{\max }$ at the third stage which reflects the distribution volume of PG in the dermis and the value of $T_{\text {sat }}$ at which PG is saturated in the dermis were calculated, and the results suggested that both the distribution volume of PG in the dermis and the time of the saturation varied depending on the enhancer. In conclusion, our present work indicated the importance and necessity of evaluating the rate and extent of appearance of a drug in the dermis to characterize an enhancer. The dermis was also suggested to act as the skin barrier to drug penetration.
\end{abstract}

Key words dermis; fatty acid; fatty amine; Azone; propylene glycol; Fourier transform infrared/attenuated total reflection (FT-IR/ATR)

Numerous skin penetration enhancers (hereafter, the enhancers) have been investigated in an attempt to deliver poorly absorbed drugs into the skin and/or systemic circulation through the stratum corneum. These enhancers are believed to reduce the skin resistance to drug penetration through the stratum corneum by disordering the tightly packed stratum corneum lipid structures and/or by altering the conformation of keratinized proteins and their associated water molecules in the corneocytes. ${ }^{1)}$ Many techniques have been used to evaluate the action of the enhancers, and many permeation studies have been made through the human and animal skins in vitro using Frantz cells. With this technique, however, the enhancers can only be characterized by the drug behavior, such as drug flux through the skin which appears in the reservoir. Another technique to examine the action of an enhancer is to measure structural changes of the stratum corneum. These are the techniques used to date to study the drug penetration mechanism. Drug behavior in the dermis, however, has not been studied except in our previous report. ${ }^{2)}$ Using Fourier transform infrared/attenuated total reflection (FT-IR/ATR) technique to pursue the behavior of drug in the dermis: (1) we will be able to learn the behavior of the enhancer in the stratum corneum from these data, (2) and also can measure how the enhancer acts on the dermal structures, which would provide information on the role of the dermis as a skin barrier to drug penetration. Therefore, such data would be different from others reported to date, and can be of value to elucidate the action of enhancers on the skin associated with their mechanisms.

* To whom correspondence should be addressed.
Fatty acids are well-known to be compounds which accelerate the penetration of polar and/or moderately polar molecules. ${ }^{3)}$ It was also reported that the skin permeability enhancing action of fatty acids was increased in coexistence with propylene glycol $(\mathrm{PG}){ }^{4,5)}$ Similar observations were reported by several researchers. ${ }^{6-9)}$ The rate of skin permeation of $P G$ could influenced the permeation rate of dissolved solutes. ${ }^{10)}$ Thus, permeation of a solvent such as PG must be an important factor influencing the solute transport. Based on these findings, the behavior of PG in the skin was viewed as a good index to characterize the enhancers when they were concomitantly administered. In our previous study, we described that the reorganization of the lipid domains due to the lipid extraction by oleic acid which dissolved in PG helped the PG molecules enter the dermis, and that an effective volume within the stratum corneum for solutes and/or solvent which could penetrate the barrier was altered in conjunction with the structural changes of the lipids. $\left.{ }^{2}\right)$

Our present work thus examined the time profile of appearance of PG in the dermis when PG was applied to rat abdominal intact skin surface together with the enhancer (e.g. fatty acids or fatty amines). The data were obtained by using FT-IR/ATR spectroscopy.

\section{MATERIALS AND METHODS}

Materials Lauric and oleic acids, and lauryl and oleylamines were purchased from Nakarai Tesque: all were of a reagent grade. Azone was generously supplied by the Nelson Research Center. PG was obtained from 
Nakarai Tesque. All other chemicals were also of a reagent grade.

Skin Preparation for FT-IR/ATR Spectroscopy Measurement As previously reported, ${ }^{11)}$ the abdominal skin was removed from male Wistar rats ( 8 - 9 weeks old) under pentobarbital anesthesia, shaved with electric clippers and then with an electric razor. The freshly excised full thickness skin with subcutaneous fat removed was weighed. The skin surface area available for FT-IR/ATR measurement was $1.05 \mathrm{~cm}^{2}$. The skin samples were then mounted between the two compartments of the diffusion cells with the dermis side facing the receiver compartment. The formulations used were PG with or without an enhancer in the concentration range between 0 and $0.15 \mathrm{M}$. One gram of the vehicle was applied into the donor compartment, and this compartment was then sealed from the atmosphere with Parafilm ${ }^{\circledR}$.The reservoir compartment was filled with $14.2 \mathrm{ml}$ of a phosphate buffered solution (PBS; $140 \mathrm{~mm} \mathrm{NaCl}, 2.68 \mathrm{mM} \mathrm{KCl}, 8.10 \mathrm{~mm}$ $\mathrm{Na}_{2} \mathrm{HPO}_{4}$ and $1.47 \mathrm{~mm} \mathrm{KH} \mathrm{PO}_{4}, \mathrm{pH}$ 7.4). The assembled diffusion cells were then immediately immersed in a water bath at $37^{\circ} \mathrm{C}$ and stirred with a magnetic stirrer. The receiver compartments were maintained at $37^{\circ} \mathrm{C}$. Each of the skin samples was removed from the cell at appropriate intervals after the incubation. The surface of the dermis tissues was gently wiped with Kimwipes ${ }^{\circledR}$ and then kept it as it was for $10 \mathrm{~min}$ at ambient temperature for FTIR/ATR measurement. Following treatment, the skin was placed, dermal surface down, on the reflection element; then spectra of the dermal surface were obtained.

FT-IR/ATR Spectroscopy Measurement IR spectra of the surface of the dermis tissues were obtained at an ambient temperature with a JEOL JIR-100 FT-IR spectrometer equipped with a liquid nitrogen cooled, narrow band mercury-cadmium-telluride detector (MCT detector) with a resolution of $0.45 \mathrm{~cm}^{-1}$. The internal reflection element was KRS-5 $(52 \times 20 \times 2 \mathrm{~mm}$ trapezoidal cut at $45^{\circ}$ ). This element permitted an IR beam to penetrate to a depth of between $0.58 \mu \mathrm{m}\left(4000 \mathrm{~cm}^{-1}\right)$ and $5.8 \mu \mathrm{m}$ $\left(400 \mathrm{~cm}^{-1}\right)$ into the dermis.

Data Analysis The time profile of the spectral peak of PG was constructed by plotting the mean spectral peak area against time. The increased PG spectral peak area (PG peak area) against time was not time dependent and seemed to consist of three phases. At the first stage, PG penetrated gradually into the dermis. At the second stage, penetration was markedly accelerated, and the penetration enhancing effect of the enhancer was thought to reach maximum (the effect was saturable). In this context, the line of the slope was regarded as being linear. At the third stage, PG was saturated in the dermis. The time to reach the maximal alteration of the dermal structure, $T_{\max \text { alteration }}$, was then obtained at the intercept on the time axis by extrapolating the linear slope of the mean value of PG peak area-time profile at the second stage using 3 to 4 data points by a regression analysis. The peak area, $(A U C)$, was calculated using a built-in programmed method equipped with the FT-IR/ATR instrument. The PG peak area $a_{\max }$ was also calculated using 3 to 5 data points after the PG spectral peak intensity became constant. The time at which PG was saturated in the dermis, $T_{\text {sat }}$, was calculated as well, and this value was determined as the time of the intersection between the slope of the plot described above and the calculated straight line of the PG peak area ${ }_{\max }$.

\section{RESULTS AND DISCUSSION}

As previously reported, ${ }^{2)} \mathrm{PG}$ appeared in the dermis following its application with $0.15 \mathrm{M}$ oleic acid on rat intact skin surface. Figure 1 shows the representative spectra of the effect of oleic acid concentrations on PG transport into the dermis. As seen, the spectral peak near $1050 \mathrm{~cm}^{-1}$, which originated from PG molecules, was not observed either with the sample of 0.01 or $0.05 \mathrm{M}$ oleic acid in PG after $2 \mathrm{~h}$-treatment of the intact skin surface. When PG was applied to the intact skin surface without any additives, PG did not give any significant peak in the spectrum after $2 \mathrm{~h}$-, or even after $10 \mathrm{~h}$-treatment (the latter data not shown). We also examined if PG and oleic acid did not interfere with each other in their spectra at the required wavenumbers (Fig. 2A and B). Based on these data, it was suggested that oleic acid did not reduce any resistance of the skin barriers at lower concentrations such as 0.05 and $0.01 \mathrm{M}$ in PG, at least for the $2 \mathrm{~h}$-treatment. At both the higher concentrations of 0.15 and $0.5 \mathrm{M}$ oleic acids, the spectral peak resulting from PG molecules was clearly visible, and the effect of oleic acid on the structure of the skin barrier was found to be concentration dependent.

Figure 3A illustrates the typical spectra of PG appearing in the dermis with time after treatment of the intact skin surface with $0.15 \mathrm{M}$ oleic acid in PG. The time profile of the mean PG peak area is shown in Fig. 3B. As seen in Fig. 3A, the PG spectral peak was not detected in the dermis with the skin sample of 5 min-treatment. However, it appeared after 15 min-treatment and increased with time. After the 30 min-treatment the spectral peak intensity

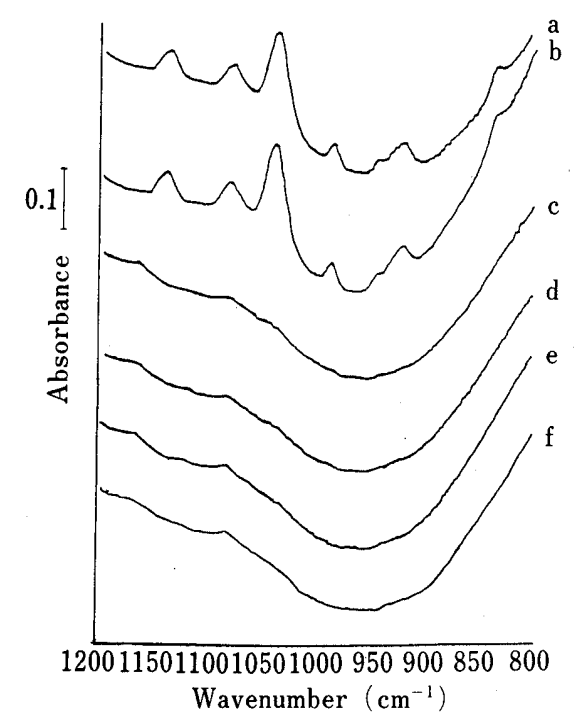

Fig. 1. Effect of Oleic Acid Concentrations on the Appearance of PG in the Dermis

Intact skin surface was treated with different concentrations of oleic acid in PG for $2 \mathrm{~h}$. $\mathrm{PG}$ was determined by measuring the spectra in the $\mathrm{C}-\mathrm{O}$ stretching region originating from the alcoholic group in the molecules. (a), $0.5 \mathrm{M}$; (b), $0.15 \mathrm{M}$; (c), $0.05 \mathrm{M}$; (d), $0.01 \mathrm{M}$; (e), PG alone; (f), no treatment. The representative spectra are shown here. Five experimnts were made with each concentrations. 


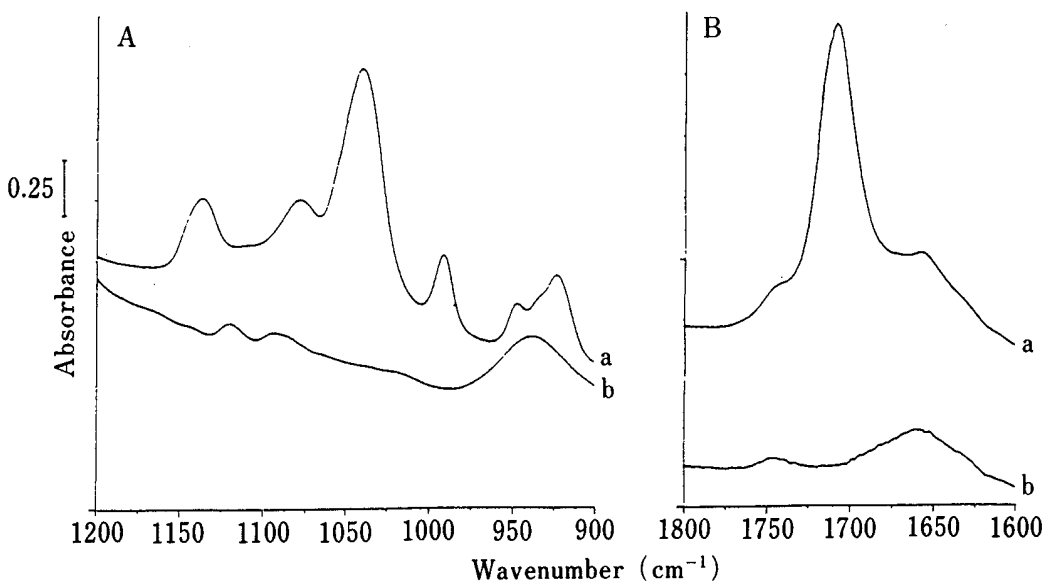

Fig. 2. Representative FT-IR Spectra of PG and Oleic Acid Pure Samples

A, only the region showing C-O stretching originating from PG molecules is depicted. (a) Spectrum for PG; (b) spectrum for oleic acid. B, only the region showing C-H stretching originating from oleic acid. (a) Spectrum for oleic acid; (b) spectrum for PG. Three experiments were made with each sample.
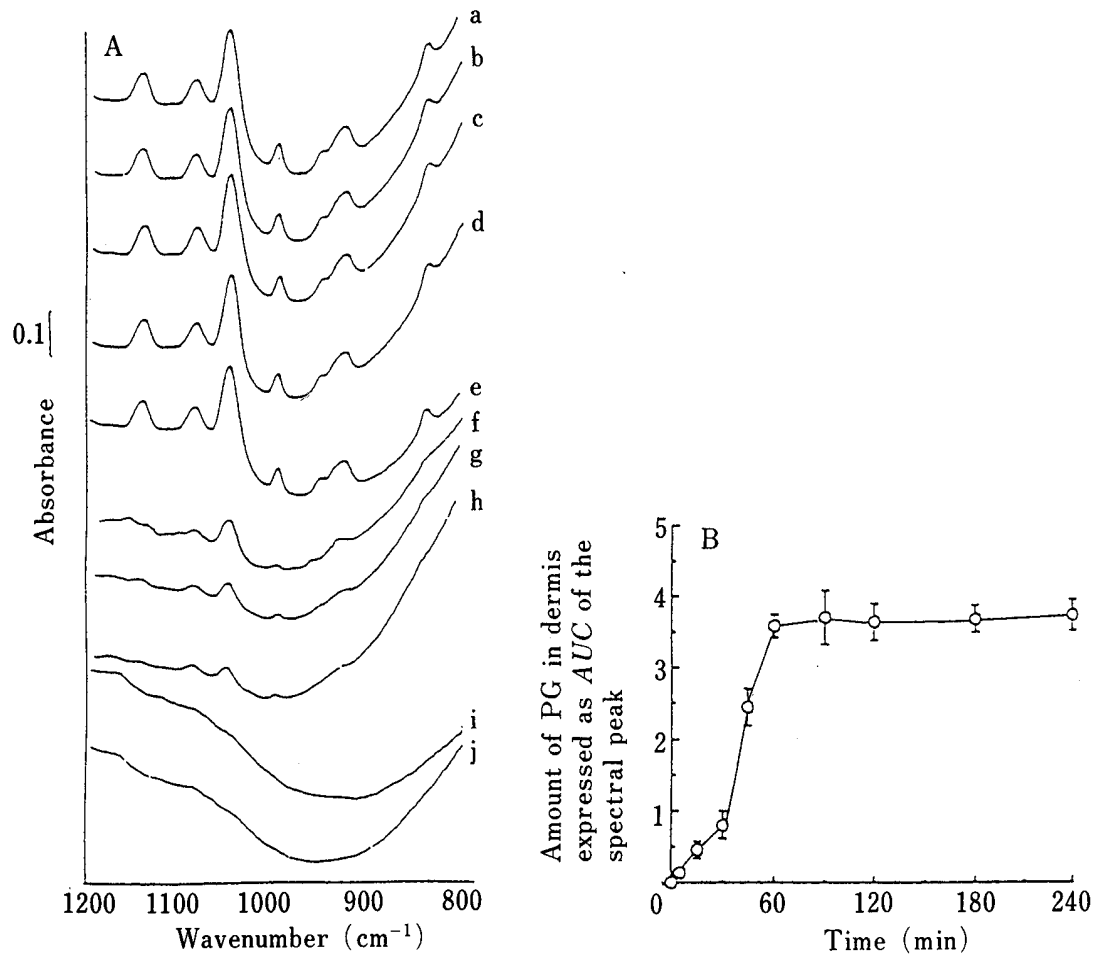

Fig. 3. Appearance of PG in the Dermis with Time after the Treatment of Rat Intact Skin Surface with $0.15 \mathrm{M}$ Oleic Acid in PG

A, representative FT-IR spectra of PG determined at (a), $4 \mathrm{~h}$; (b), $3 \mathrm{~h}$; (c), $2 \mathrm{~h}$; (d), $90 \mathrm{~min}$; (e), $60 \mathrm{~min}$; (f), $45 \mathrm{~min}$; (g), $30 \mathrm{~min}$; (h), $15 \mathrm{~min}$; (i), $5 \mathrm{~min}$; (j), without oleic acid treatment. B, time profile of the amount of PG appearing in dermis expressed as the peak area of the FT-IR spectrum of PG. Each value represents the mean \pm S.E. $(n=26)$.

increased markedly as apparent in Fig. 3B. When the time of the treatment exceeded more than $1 \mathrm{~h}$, the intensity of the PG spectral peak seemed to reach maximum and constant. Since PG itself did not almost penetrate the skin barrier and was not distributed in the dermis in the absence of any additive, oleic acid was considered to facilitate the entrance of PG into the dermis so that the volume there possibly increased. Thus it is suggested that the reduction in barrier resistance of the skin by oleic acid proceeded gradually at the initial stage, and at the second stage the rapid distribution of $P G$ molecules into the dermis occurred followed by the saturation in the dermis. This rapid distribution believed to be caused by alteration of the dermal structure.

As to the lag time until PG penetrated through an entire rat skin, Yamada et al. ${ }^{5)}$ showed in their article that approximately $2 \mathrm{~h}$ was required for PG permeation through the excised whole skin when $10 \%$ oleic acid in PG (equivalent to $0.35 \mathrm{M}$ oleic acid) was applied to the intact skin surface using two compartment diffusion cells. This reported lag time was much greater than our value of 15 min at which PG appeared in the dermis (at the region adjacent to the solution in the reservoir). The time PG appeared was actually not that when it was detected in the reservoir. However, it would be rational to consider the time of appearance in the dermis as the approximate time of penetration of PG molecules through the whole skin, since we measured PG appearing at the interface between the dermis and the solution. The above described difference in the time of PG penetration through the whole 
skin could/can thus probably be attributed to the difference in the site of the determination. One possible explanation for such difference is that the distribution rate into the dermis was faster than the transfer rate into the reservoir.

As for the action of oleic acid on the dermal structure, the permeation profile at the second stage using the peak areas for 30, 45 and $60 \mathrm{~min}$ was constructed against time. Then, the value of $T_{\max a l t e r a t i o n}$ was obtained at the intercept on the time axis by extrapolating from the straight line. This calculated value was $13 \mathrm{~min}$ as shown in Table I $a$ ). From this value, it was presumed that oleic acid maximally altered the dermal structures within at least $13 \mathrm{~min}$. When lauric acid was at the concentration of $0.15 \mathrm{M}$ in PG, the spectral peak was slightly detected with the 30 min-treated sample (Fig. 4A, B), but this peak height was only about $30 \%$ of that for the 30 min-oleic acidtreated one. $T_{\max \text { alteration }}$ for lauric acid was $131 \mathrm{~min}$ and was clearly longer than that for oleic acid $(13 \mathrm{~min}),(p<$

TABLE I. Parameters Characterizing Skin Penetration Enhancers

\begin{tabular}{lcccr}
\hline \hline $\begin{array}{c}\text { Skin } \\
\text { penetration } \\
\text { enhancer }\end{array}$ & $\begin{array}{c}T_{\text {max alteration }}{ }^{a)} \\
(\mathrm{min})\end{array}$ & Slope ${ }^{b)}$ & $\begin{array}{c}\text { PG peak } \\
\text { area }_{\text {max }}{ }^{c}\end{array}$ & \multicolumn{1}{c}{$\begin{array}{c}T_{\text {sat }}{ }^{d} \\
(\text { min) }\end{array}$} \\
\hline Oleic acid & $13.2^{e)}$ & $0.0729^{())}$ & $3.67 \pm 0.50^{e)}$ & 63.1 \\
Lauric acid & $131.4^{f)}$ & $0.0085^{f)}$ & $1.95 \pm 0.70^{f)}$ & 358.6 \\
Oleylamine & $12.3^{g)}$ & $0.0793^{g)}$ & $4.99 \pm 0.71^{g)}$ & 75.3 \\
Laurylamine & $169.1^{h)}$ & $0.0254^{h)}$ & $3.75 \pm 0.78^{h)}$ & 316.7 \\
Azone & $123.3^{i)}$ & $0.0087^{i)}$ & $5.27 \pm 1.08^{i)}$ & 425.3 \\
\hline
\end{tabular}

The values were obtained by treatment of the rat abdominal intact skin surface with $0.15 \mathrm{M}$ skin penetration enhancer in PG. The values of PG peak area ${ }_{\max }$ are mean \pm S.E. Other parameters are the mean values. All values were obtained by the method described in the experimental section (data analysis). Numbers of trials were 26 for oleic acid-, 17 for lauric acid-, 15 for both laurylamine- and oleylamineand 20 for Azone-treated sample. Statistical significant differences were determined by Student's $t$-test. The significance was as follows: a) $e$ and $c$ were significantly different from $f, h$, and $i$, respectively, $(p<0.001)$. $f$ was significantly different from both $h$ and $i,(p<0.001) . h$ was significantly different from $i,(p<0.001) . e$ was not significantly different from $g . \quad b$ ) The values were significantly different from each other except between $e$ and $g$, and between $f$ and $i,(p<0.001) . c) e$ was significantly different from $f, g$, and $i,(p<0.001)$. $f$ was significantly different from $g, h$, and $i$, $(p<0.001) . h$ was significantly different from $i,(p<0.001)$. Others were not significantly different from each other.

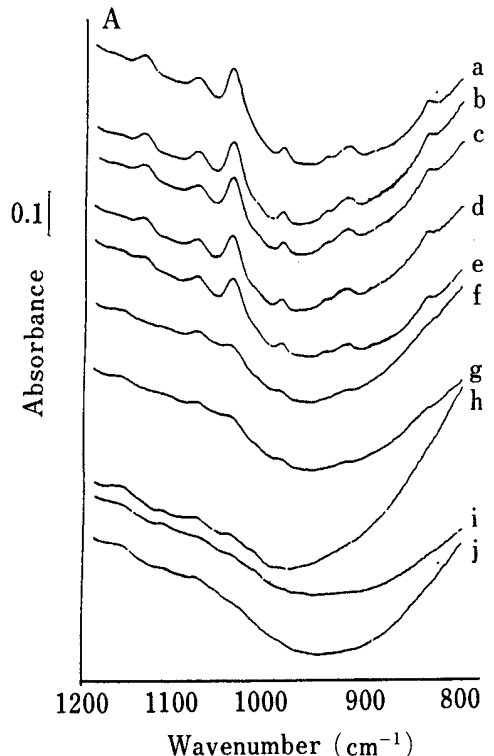

0.001 ). The value of the slope with lauric acid was about one tenth that for oleic acid as shown in Table $\mathrm{I} b$ ), $(p<0.001)$. Thus, both these results confirmed that lauric acid was significantly slower than oleic acid in producing the maximal alteration of the structures. Once the enhancer reduces the barrier resistance by inducing the structural alteration of lipids and proteins of the skin, a substance or drug can easily distribute in the skin, and the amount of the substance incorporated into the skin is presumably increased. We therefore compared the dermal distribution volume of PG between each of the enhancers with the values of PG peak area $\max _{\max }$ (Table Ic). The data showed that the mean PG level in dermis followed by the lauric acid-treatment was definitely lower than in the case of the oleic acid; the value was almost one half. Therefore, both oleic acid and lauric acid were effective in increasing the dermal distribution volume by their structural alteration effect, but the action of lauric acid was significantly the weaker of the two, $(p<0.001)$. As shown in Table I $d), T_{\text {sat }}$ for oleic acid and lauric acid were 63.1 and $358.6 \mathrm{~min}$, respectively. This meant that PG was more rapidly saturated in the dermis with the oleic acid-treated sample than in the dermis with the lauric acid.

Figures $5 \mathrm{~A}$ and $\mathrm{B}$ illustrate the results with $0.15 \mathrm{M}$ oleylamine. The PG spectral peak appeared very slightly at $5 \mathrm{~min}$ after the initiation of the treatment, and became more distinct at $15 \mathrm{~min}$. The results of oleylamine and oleic acid in both $T_{\max \text { alteration }}$ and the slope were similar as indicated in Table I $e$ ) and $g$ ). Thus, the dermal structure was maximally disordered within about the same time by both substances. However, the PG peak area $_{\max }$ for the oleylamine-treated sample was about 1.3 times the value for the oleic acid-treated one $(p<0.001)$ as shown in Table Ic), so that oleylamine was found to be more effective in increasing the dermal distribution volume of the dermis.

In the case of laurylamine, the representative spectra of PG are illustrated in Fig. 6A. Appearance of PG was not distinct at the initial stage, and after the $3 \mathrm{~h}$-treatment its presence in the dermis was notable. From the results in

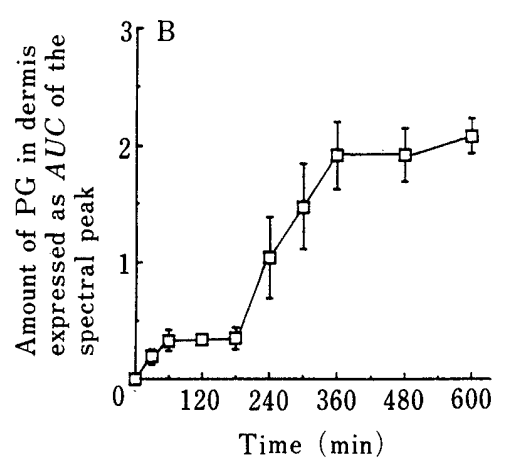

Fig. 4. Appearance of PG in the Dermis with Time after the Treatment of Rat Intact Skin Surface with $0.15 \mathrm{M}$ Lauric Acid in PG

A, representative FT-IR spectra of PG determined at (a), $10 \mathrm{~h}$; (b), $8 \mathrm{~h} ;(\mathrm{c}), 6 \mathrm{~h}$; (d), $5 \mathrm{~h} ;(\mathrm{e}), 4 \mathrm{~h} ;(\mathrm{f}), 3 \mathrm{~h} ;(\mathrm{g}), 2 \mathrm{~h} ;(\mathrm{h}), 60 \mathrm{~min}$; (i), 30 min; (j), without lauric acid treatment. B, time profile of the amount of PG appearing in dermis expressed as the peak area of the FT-IR spectrum of PG. Each value represents the mean \pm S.E. $(n=17)$. 

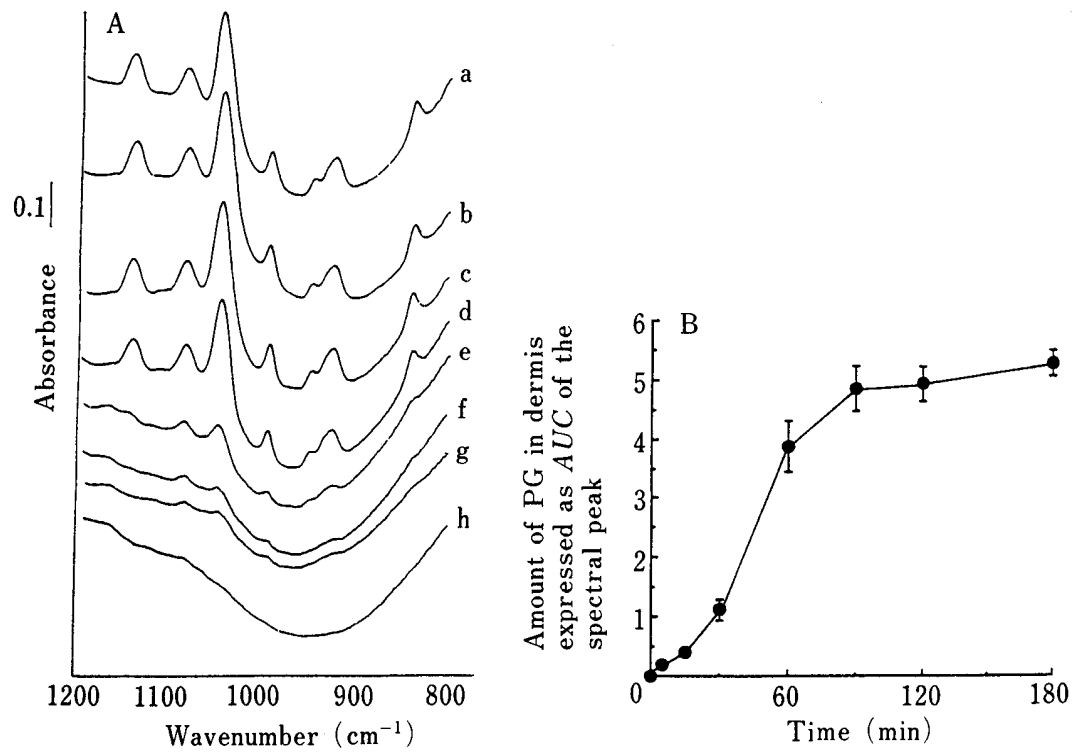

Fig. 5. Appearance of $P G$ in the Dermis with Time after the Treatment of Rat Intact Skin Surface with $0.15 \mathrm{M}$ Oleylamine in PG

A, representative FT-IR spectra of PG determined at (a), $3 \mathrm{~h}$; (b), $2 \mathrm{~h}$; (c), $90 \mathrm{~min}$; (d), $60 \mathrm{~min}$; (e), $30 \mathrm{~min}$; (f), $15 \mathrm{~min}$; (g), $5 \mathrm{~min}$; (h), without oleylamine treatment. B, time profile of the amount of PG appearing in dermis expressed as the peak area of the FT-IR spectrum of PG. Each value represents the mean \pm S.E. $(n=15)$.
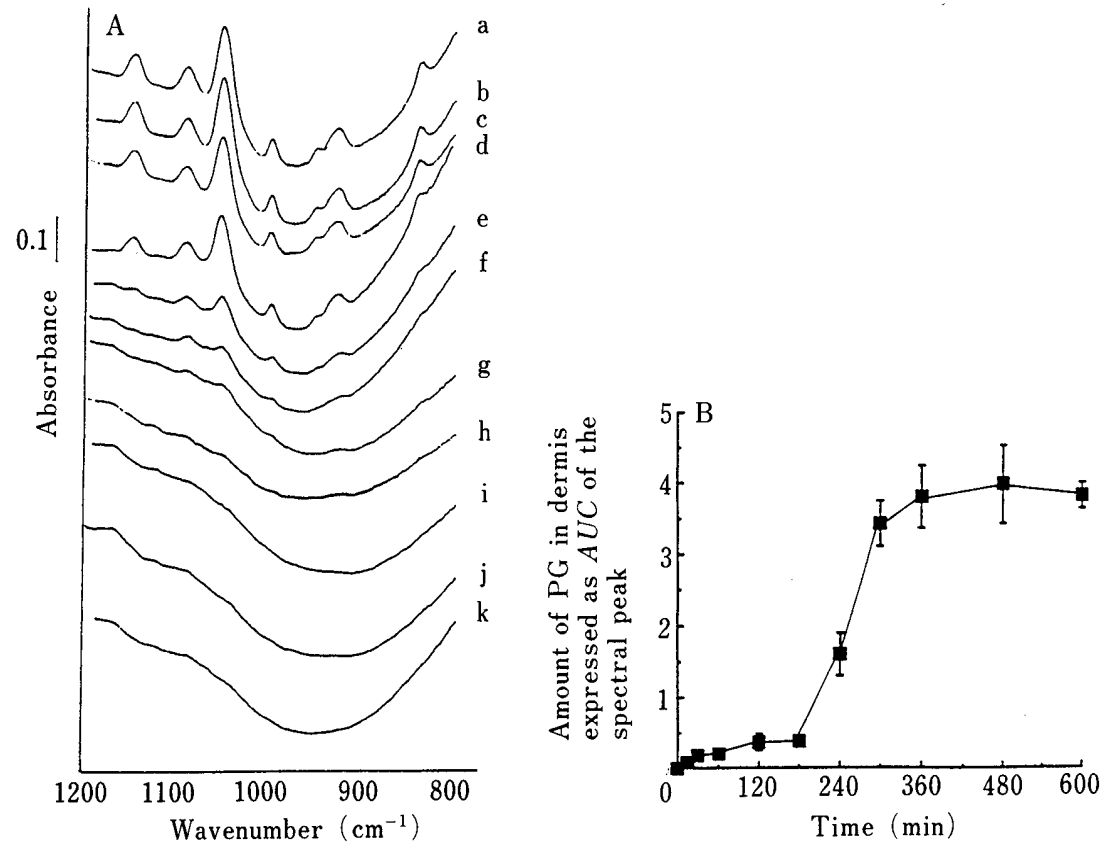

Fig. 6. Appearance of PG in the Dermis with Time after the Treatment of Rat Intact Skin Surface with $0.15 \mathrm{M}$ Laurylamine in PG

A, representative FT-IR spectra of PG determined at (a), $10 \mathrm{~h}$; (b), $8 \mathrm{~h}$; (c), $6 \mathrm{~h}$; (d), $5 \mathrm{~h} ;(\mathrm{e}), 4 \mathrm{~h}$; (f), $3 \mathrm{~h} ;(\mathrm{g}), 2 \mathrm{~h}$; (h), $60 \mathrm{~min} ;(\mathrm{i}), 30 \mathrm{~min} ;(\mathrm{j}), 15 \mathrm{~min} ;(\mathrm{k})$, without laurylamine treatment. B, time profile of the amount of PG appearing in dermis expressed as the peak area of the FT-IR spectrum of PG. Each value represents the mean \pm S.E. $(n=20)$.

Fig. $6 \mathrm{~B}, \mathrm{~T}_{\max \text { alteration }}$ was calculated and the value was about $170 \mathrm{~min}$ as indicated in Table $\mathrm{I} a$ ). This was definitely larger than that for oleylamine $(13 \mathrm{~min}),(p<0.001)$, suggesting that laurylamine was less potent in enhancing the penetration of PG through the stratum corneum and the epidermal barriers. When $T_{\max \text { alteration }}$ was compared between laurylamine and lauric acid, which have the same number of carbon of alkylchain in their molecules, the former was about $40 \mathrm{~min}$ slower in causing the structural alteration of the dermis than the latter $(p<0.001)$. However, laurylamine increased the rate of distribution of PG in the dermis as compared with lauric acid, since the value of the slope was larger with the former than the latter, while $T_{\text {sat }}$ of the two was similar (Table I). These results might lead to a possible conclusion that both amino and carboxyl groups are factors accelerating the penetration of PG into the dermis. Further experiment are required to elucidate details as to the degree of their contribution to the accelerating action. It is also of interest that laurylamine increased the dermal distribution volume about twice as much as lauric acid, since the PG peak area $_{\max }$ for laurylamine was almost twice that for lauric acid $(p<0.001)$ as shown in Table $\mathrm{I} c)$.

A well-known enhancer, Azone was tested and its action compared with those of fatty acids and fatty amines. As seen in Fig. 7, the spectrum originated from PG was hardly 

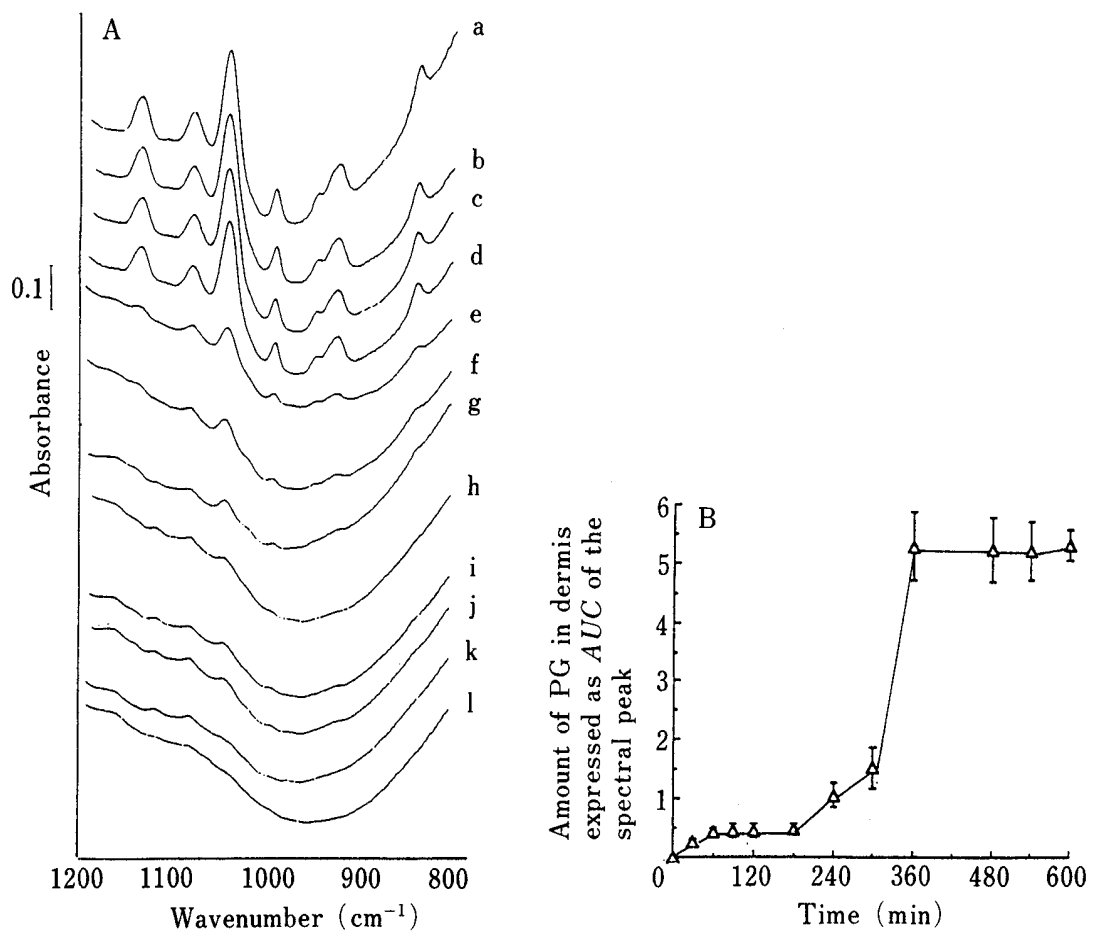

Fig. 7. Appearance of PG in the Dermis with Time after the Treatment of Rat Intact Skin Surface with $0.15 \mathrm{M}$ Azone in PG

A, representative FT-IR spectra of PG determined at (a), $10 \mathrm{~h}$; (b), $9 \mathrm{~h} ;(\mathrm{c}), 8 \mathrm{~h} ;(\mathrm{d}), 6 \mathrm{~h} ;(\mathrm{e}), 5 \mathrm{~h} ;(\mathrm{f}), 4 \mathrm{~h} ;(\mathrm{g}), 3 \mathrm{~h} ;(\mathrm{h}), 2 \mathrm{~h}$; (i), $90 \mathrm{~min} ;(\mathrm{j}), 60 \mathrm{~min} ;(\mathrm{k}), 30 \mathrm{~min}$; (l), without Azone treatment. B, time profile of the amount of PG appearing in dermis expressed as the peak area of the FT-IR spectrum of PG. Each value represents the mean \pm S.E. $(n=24)$.

observed at $30 \mathrm{~min}$. Nor did further treatment produce a remarkable PG spectral peak in the dermis as was observed with the 1-, 1.5- and $2 \mathrm{~h}$-treated samples. The penetration of PG was, indeed, very slow and the remarkable spectral peak did not appear until $6 \mathrm{~h}$. This peak intensity was almost equal to that for the 90 min-treated sample of oleylamine. Appearance of $\mathrm{PG}$ in the dermis with the Azone was therefore retarded. As shown in Table I, $T_{\max \text { alteration }}$ for Azone was $123 \mathrm{~min}$, much larger than that for oleic acid $(13 \mathrm{~min})$ or for oleylamine $(12 \mathrm{~min})$. The value of the slope with Azone was quite similar to lauric acid, and PG peak area $a_{\max }$ was the largest on average among the skin penetration enhancers used. Azone was reported to increase retention of water in the lipid matrix of the stratum corneum by penetrating the lamella bilayer, and also to provide a potential hydrophobic region which produced an enhancement of permeation of hydrophobic molecules. ${ }^{12)}$ This characteristic of Azone allows PG molecules, a surfactant like compound with the molecular weight of 76.09 , to easily incorporate into the lipid bilayers of the stratum corneum and then more transfer into the dermis. However, as described above, PG permeation rate into the dermis was slower than with oleic acid and oleylamine, and Azone was similar to laurylamine in its behavior. Based on these results, it is rational to think that Azone can stay in the stratum corneum lipid bilayers due to its strong affinity with the lipid molecules, and can migrate very slowly into the dermis from the stratum corneum and thus cause retardation of PG transfer into the dermis.

In conclusion, the appearance of PG caused by skin penetration enhancers with time seems to occur in three phases. It is believed that (1) at the first stage, PG penetrates through the skin barriers which are not substantially disordered and gradually appears in the dermis; (2) at the second stage, it rapidly distributes in/throughout the dermis because of changes in the dermal structure: the penetration enhancing effect of the enhancer was thought to reach maximal; and (3) at the third stage it is saturated in the dermis. Our findings suggest that both the rate of the dermal structural alteration and the size of the distribution volume in the dermis varied with skin penetration enhancers. Our data thus offer an indication of the importance and necessity of evaluating the rate and extent of appearance of a compound in the dermis in characterizing skin penetration enhancers.

\section{REFERENCES}

1) B. W. Barry, J. Control Rel., 6, 85 (1987).

2) Y. Takeuchi, H. Yasukawa, Y. Yamaoka, N. Takahashi, T. Tamura, Y. Morimoto, S. Fukushima, R. C. Vasavada, Chem. Pharm. Bull., 41, 1434 (1993).

3) B. Ongpipattanakul, R. R. Burnette, R. O. Potts, M. L. Francoeur, Pharm. Res., 8, 350 (1991).

4) B. J. Aungst, N. J. Rogers, E. Sheffter. Int. J. Pharm., 33, 225 (1986).

5) M. Yamada, Y. Uda, Y. Tanigawara, Chem. Pharm. Bull., 35, 3399 (1987).

6) B. Mollgaard, A. Hoelgaard, Acta Pharm. Suec., 20, 443 (1983).

7) F. Kaiho, H. Nomura, E. Makabe, Y. Kato, Chem. Pharm. Bull., 35, 2928 (1987)

8) M. Mahjour, B. E. Mauser, M. B. Fawzi, Int. J. Pharm., 56, 1 (1989).

9) K. Sato, K. Sugibayashi, Y. Morimoto, Int. J. Pharm., 43, 31 (1988).

10) B. Aungst, J. A. Blake, M. A. Hussain, Pharm. Res., 7, 712 (1990).

11) Y. Takeuchi, H. Yasukawa, Y. Yamaoka, Y. Kato, Y. Morimoto, Y. Fukumori, T. Fukuda, Chem. Pharm Bull., 40, 1887 (1992).

12) A. J. I. Ward, R. Tallon, Drug Dev. Ind. Pharm., 14, 1155(1988). 